\title{
Flat Detector Angio-CT following Intra-Arterial Therapy of Acute Ischemic Stroke: Identification of Hemorrhage and Distinction from Contrast Accumulation due to Blood-Brain Barrier Disruption
}

T. Kau, M. Hauser, S.M. Obmann, M. Niedermayer, J.R. Weber, and K.A. Hausegger

\begin{abstract}
BACKGROUND AND PURPOSE: Flat panel detector CT in the angiography suite may be valuable for the detection of intracranial hematomas; however, abnormal contrast enhancement frequently mimics hemorrhage. We aimed to assess the accuracy of flat panel detector CT in detecting/excluding intracranial bleeding after endovascular stroke therapy and whether it was able to reliably differentiate hemorrhage from early blood-brain barrier disruption.
\end{abstract}

MATERIALS AND METHODS: Seventy-three patients were included for retrospective evaluation following endovascular stroke therapy: 32 after stent-assisted thrombectomy, 14 after intra-arterial thrombolysis, and 27 after a combination of both. Flat panel CT images were assessed for image quality and the presence and type of intracranial hemorrhage and BBB disruption by 2 readers separately and in consensus. Follow-up by multisection head CT, serving as the reference standard, was evaluated by a single reader.

RESULTS: Conventional head CT revealed intracranial hematomas in 12 patients (8 subarachnoid hemorrhages, 7 cases of intracerebral bleeding, $3 \mathrm{SAH}$ s plus intracerebral bleeding). Image quality of flat panel detector CT was considered sufficient in all cases supratentorially and in $92 \%$ in the posterior fossa. Regarding detection or exclusion of intracranial hemorrhage, flat panel detector CT reached a sensitivity, specificity, positive and negative predictive values, and accuracy of $58 \%, 85 \%, 44 \%, 91 \%$, and $81 \%$, respectively. Maximum attenuation measurements were not valuable for the differentiation of hemorrhage and BBB disruption.

CONCLUSIONS: Flat panel CT after endovascular stroke treatment was able to exclude the rare event of an intracranial hemorrhage with a high negative predictive value. Future studies should evaluate the predictive value of BBB disruptions in flat panel detector CT for the development of relevant hematomas.

ABBREVIATIONS: $\mathrm{CCT}=$ conventional $\mathrm{CT} ; \mathrm{FPCT}=$ flat panel detector $\mathrm{CT} ; \mathrm{ICB}=$ intracerebral bleeding; $\mathrm{PH}=$ parenchymal hematoma

$F^{a}$ at panel detectors in modern angiographic C-arm systems allow almost instant access to CT-like cranial imaging in the angiography suite. ${ }^{1-4}$ While flat panel detector CT (FPCT) is currently not reliable in depicting ischemic brain lesions, it proved to be a sensitive tool for the detection of intracranial hematomas in both experimental and clinical settings. ${ }^{5,6}$ However, there have been numerous reports of abnormal contrast enhancement following neurovascular interventions that frequently mimic subarachnoid, intraventricular, or intracerebral hemorrhage..$^{7-9}$ It is important to recognize such patterns of temporary blood-brain

Received December 13, 2013; accepted after revision February 18, 2014. From the Institute of Diagnostic and Interventional Radiology (T.K., M.H., M.N., K.A.H.) and Department of Neurology (S.M.O., J.R.W.), Klinikum Klagenfurt, Klagenfurt, Austria.

Please address correspondence to Thomas Kau, MD, Klinikum Klagenfurt, Institute of Diagnostic and Interventional Radiology, Feschnigstr 11, 9020 Klagenfurt, Austria; e-mail: t.kau@gmx.at

三 Indicates article with supplemental on-line table.

http://dx.doi.org/10.3174/ajnr.A4021 barrier disruption in postprocedural imaging because misinterpretation may unnecessarily delay anticoagulant/antiaggregant treatment.

In the present study, we aimed to assess the diagnostic accuracy of FPCT for the detection of intracranial bleeding immediately after endovascular stroke therapy and whether it was able to reliably differentiate hemorrhage from early blood-brain barrier disruption.

\section{MATERIALS AND METHODS}

The study was approved by our local ethics commission.

\section{Subjects}

Between May 2008 and December 2011, 100 endovascular stroke interventions had been performed in our institution. Postinterventional FPCT was not available in 16 patients. Another 11 patients were excluded from further assessment due to a lack of early conventional CT (CCT) follow-up as defined below. Finally, 73 patients were included in this retrospective study.

AJNR Am J Neuroradiol 35:1759-64 Sep 2014 www.ajnr.org 

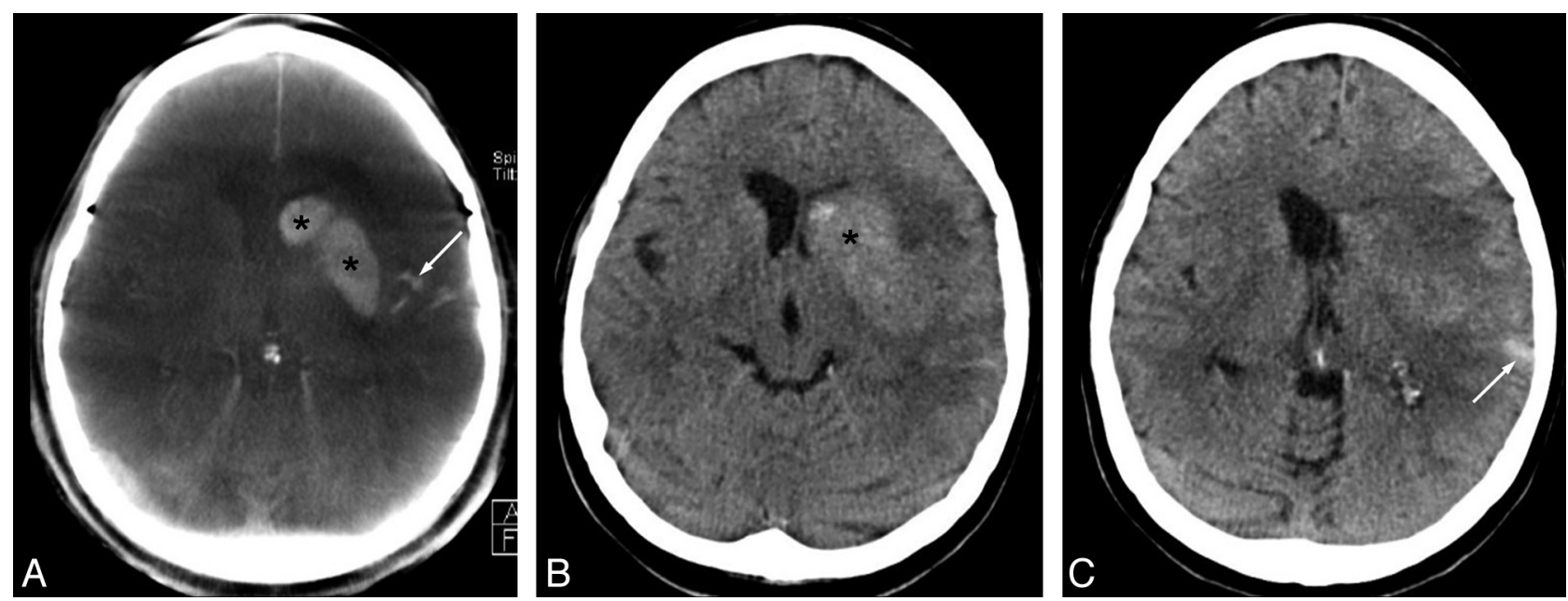

FIG 1. Blood-brain barrier disruption (asterisks) and subarachnoid hemorrhage (arrows) following combined intra-arterial thrombolysis $(1,000,000 \mathrm{U}$ of urokinase) and thrombectomy in a 62-year-old female patient with occlusion of the left middle cerebral artery (admission modified Rankin Scale score, 5; stroke time to admission uncertain; recanalization-to-follow-up CCT, 19.1 hours). Hyperattenuations in the left hemispheric sulci (SAH) sharply confined to the ipsilateral striatum (BBB disruption) are clearly visible on postinterventional FPCT (A) and confirmed by CCT (B and $C)$.

\section{Acute Stroke Therapy}

Acute stroke therapy consisted of endovascular intervention in all cases with 7 patients (10\%) having received a "bridging" dose of recombinant tissue plasminogen activator intravenously in advance. Thirty-two patients (44\%) were treated by stent-assisted thrombectomy; 14 patients (19\%), by intra-arterial thrombolysis with urokinase; and 27 patients (37\%), by a combination of both. The mean dose of urokinase administered intra-arterially in 41 patients was 600,000 (range, 150,000-1,100,000) units. The technique of endovascular stroke treatment has been described elsewhere in the literature. ${ }^{10-12}$

\section{Flat Panel CT Image Acquisition and Interpretation}

Flat panel CT images were acquired on a biplane angiography system (DynaCT, Axiom Artis dBA; Siemens, Erlangen, Germany) in 64 patients with the following parameters: voltage, 95.5 $\mathrm{kV}$; tube current, $206.5 \mathrm{~mA}$; acquisition time, $12.5 \mathrm{~ms}$ per frame; projection on $30 \times 40 \mathrm{~cm}$ flat panel size; angulation, $0.4^{\circ}$ per frame; total angle, $220^{\circ}$; exposure time, 20 seconds. In 9 patients, FPCT imaging was performed on a single-plane angiography system (XperCT, Allura Xper FD20 with Xtra Vision; Philips Healthcare, Best, the Netherlands) with the following parameters: voltage, $120 \mathrm{kV}$; tube current, $250 \mathrm{~mA}$; frame speed, 30 frames per second; acquisition time, $5 \mathrm{~ms}$ per frame; total angle, $240^{\circ}$; exposure time, 20.8 seconds; FOV, $48 \mathrm{~cm}$.

Flat panel CT images were assessed for the presence of subarachnoid hemorrhage, intracerebral bleeding (ICB), and BBB disruption by 2 readers (T.K., with 9 years of experience in radiology, Austrian board-certified radiologist, European board-certified neuroradiologist; M.H., with 7 years of experience in radiology, Austrian board-certified radiologist) separately, followed by consensus reading. Both readers were aware of which vessel had been occluded initially; however, they were blinded to further clinical data and conventional CT results. Pathologic intracerebral hyperattenuations were considered the result of BBB disruption if the borders of anatomic structures or vascular territories



FIG 2. Flat panel CT immediately after stent-assisted recanalization of a carotid bifurcation occlusion with intravenous thrombolytic bridging therapy in a 72-year-old male patient (admission modified Rankin Scale score, 4; stroke time to admission, $>3$ hours; recanalization-to-follow-up CCT, 14.7 hours). Focal hyperattenuation (arrow) of the right hippocampus is believed to be due to contrast enhancement attributable to disruption of the blood-brain barrier.

were clearly respected (Fig 1). If this criterion was not applicable because of small size, the differentiation between $\mathrm{BBB}$ disruption and ICB was made at the discretion of the observer (Fig 2). According to Fiorelli et al, ${ }^{13}$ we divided hemorrhagic transformations of cerebral infarcts into 4 subtypes: hemorrhagic infarction type I, hemorrhagic infarction type II, parenchymal hematoma type I, and parenchymal hematoma type II. Subarachnoid hemorrhage was classified as suggested by Fisher et al. ${ }^{14}$ The diagnostic quality of FPCT images was assessed by both readers in consensus (sufficient versus insufficient). Supratentorial image quality was considered good if a hemorrhage or BBB disruption could be detected or excluded with high confidence. With respect to typical FPCT artifacts in the posterior fossa, infratentorial image quality was considered sufficient if a parenchymal hematoma ${ }^{13}$ and a subarachnoid hemorrhage Fisher type 3 or 4 could be detected or 



FIG 3. Correlation of image quality in an 84-year-old male patient (admission modified Rankin Scale score, 5 ; wake-up stroke, time to admission uncertain; recanalization-to-follow-up CCT, 22.2 hours). A, After mechanical recanalization of the right middle cerebral artery, FPCT shows moderate ring artifacts, however, no complication in the form of intracranial bleeding. $B$, Follow-up CCT depicts ischemic infarction of the ipsilateral putamen and no hemorrhage. C, Limited image quality of FPCT in the posterior fossa due to beam-hardening artifacts is evident.

excluded with high confidence (Fig 3). It was considered good if the readers were highly confident in detecting or excluding any hyperattenuation attributable to hemorrhage or BBB disruption.

Both readers performed maximum attenuation measurements in pathologically hyperattenuated regions with a predefined size of the region of interest $\left(10 \mathrm{~mm}^{2}\right)$.

\section{Conventional CT Image Acquisition and Interpretation}

Conventional head CT imaging was performed on a $2 \times 32$ detector row dual-source CT scanner (Somatom Definition; Siemens) on average 17.2 hours (mean, 17.5 hours; range, 0.6-45.9 hours) after FPCT. Typically, a single noncontrast CT acquisition was obtained ranging from the vertex to the craniocervical junction at an angle parallel to the skull base. Data were acquired in a caudocranial direction with the following CT parameters: pitch factor, 0.55 ; collimation, $64 \times 0.6 \mathrm{~mm}$; reconstructed section thickness, $4 \mathrm{~mm}$; FOV , $220 \mathrm{~mm}$; tube voltage and current, $120 \mathrm{kV}$ and $300 \mathrm{~mA} ; 64$ sections per rotation at a rotation time of $1 \mathrm{sec}-$ ond. Conventional CT, serving as the criterion standard for the detection and exclusion of intracranial hemorrhage, was evaluated by a single reader (K.A.H., with 26 years of experience, Austrian board-certified radiologist, European board-certified interventional radiologist). This radiologist was aware of which vessel had been occluded initially; however, he was blinded for further clinical data and FPCT results. Pathologic intracerebral hyperattenuation was thought due to $\mathrm{BBB}$ disruption if the borders of anatomic structures or vascular territories were clearly respected.

\section{Statistics}

In a first step, descriptive statistics were calculated. We used the McNemar test and $\kappa$ statistics (GraphPad Prism software; GraphPad Software, San Diego, California) to compare FPCT with CCT for its ability to detect or exclude hemorrhage in general, SAH, ICB, and BBB disruption. The degree of agreement was assessed by sensitivity, specificity, positive and negative predictive values, and accuracy, including 95\% confidence intervals (VassarStats; Vassar College, Poughkeepsie, New York). Interrater reliability was assessed by using $\kappa$ statistics. After dividing
FPCT hyperattenuation into SAH, ICB, and BBB disruptions, we used the median test to compare median values of maximum attenuation measurements in true-positive and false-positive regions. A value of $P<.05$ was considered significant. The data were analyzed by using GraphPad and VassarStats, which are freely available on-line software tools.

\section{RESULTS}

\section{Patient Characteristics}

Of 73 patients, 39 were women and 34 were men with a median age of 70 years (range, 31-85 years). The thromboembolic clot was located in the M1 segment of the middle cerebral artery in 35 patients, in the carotid bifurcation in 16 patients, in both the proximal internal carotid artery and middle cerebral artery in 4 patients, and in the basilar artery in 18 patients. Twenty-six patients were admitted to the emergency department with a modified Rankin Scale score of 4 , and 47 patients, with a score of 5 . In 38 patients (52\%), the time interval from stroke onset to initial CT was below a threshold of 3 hours. It was above 3 hours in 9 cases (12\%) and entirely uncertain in 26 patients (36\%).

\section{Image Quality}

In the supratentorial compartment, FPCT image quality was considered sufficient in all cases $(100 \%)$. Infratentorially, it was rated sufficient in 67 cases $(92 \%)$ and insufficient in the remaining $6(8 \%)$.

\section{Intracranial Hemorrhage}

Conventional CT revealed an intracranial hematoma in 12 of 73 patients (16\%). Of these, 8 subjects had SAH, and ICB was found in 7 patients. In 3 cases, both SAH and ICB were reported in the diagnostic test of reference. The On-line Table gives an overview of the findings of both readers. When comparing FPCT with CCT, the difference in their ability to detect or exclude intracranial bleeding in the follow-up of endovascular stroke treatment was found to be not statistically significant (reader $1, P=.30$; reader 2 , $P=.58)$. However, the degree of agreement proved to be only fair $(\kappa=0.36$; 95\% CI, 0.07-0.65) and moderate $(\kappa=0.41 ; 95 \%$ CI, 
Descriptive statistical data

\begin{tabular}{|c|c|c|c|c|c|c|c|c|}
\hline & \multicolumn{2}{|c|}{ Hemorrhage } & \multicolumn{2}{|c|}{ ICB } & \multicolumn{2}{|c|}{ SAH } & \multicolumn{2}{|c|}{ BBBD } \\
\hline & Reader 1 & Reader 2 & Reader 1 & Reader 2 & Reader 1 & Reader 2 & Reader 1 & Reader 2 \\
\hline True-positive & 7 & 7 & 2 & 2 & 6 & 6 & 8 & 10 \\
\hline True-negative & 51 & 53 & 58 & 64 & 61 & 57 & 53 & 50 \\
\hline False-positive & 10 & 8 & 8 & 2 & 4 & 8 & 8 & 11 \\
\hline False-negative & 5 & 5 & 5 & 5 & 2 & 2 & 4 & 2 \\
\hline
\end{tabular}

Note:-BBBD indicates blood-brain barrier disruption.

$0.12-0.70)$, respectively. Consensus reading led to 7 true-positive, 52 true-negative, 9 false-positive, and 5 false-negative results. Consequently, FPCT reached sensitivity, specificity, positive and negative predictive values, and accuracy of 58\% (range, 29\%84\%), 85\% (range, 73\%-93\%), 44\% (range, 21\%-69\%), 91\% (range, $80 \%-97 \%$ ), and $81 \%$ (7 range, $0 \%-88 \%$ ). For descriptive statistical data, see the Table .

\section{Type of Hemorrhage}

While CT could exclude SAH in 65 cases (Fisher score 1) following stroke intervention, 3 patients were assigned a score of 2 , two were assigned a score of 3 , and 3 were assigned the highest Fisher score of 4 (Fig 1). Regarding hemorrhagic transformation of ischemic brain lesions, CT revealed hemorrhagic infarction type II in 4 patients. Parenchymal hematoma was found in 3 patients ( 1 type I and 2 type II), all of which showed a combination of ICB and SAH.

\section{Blood-Brain Barrier Disruption}

In 12 patients $(16 \%)$, disruption of the BBB was found on CCT (Fig 1). With moderate agreement between FPCT and CCT, the difference in their diagnostic ability to detect or exclude BBB disruption was shown to be significant $(P=.03)$ by 1 reader. The sensitivity, specificity, positive and negative predictive values, and accuracy for this feature were 75\% (range, 43\%-93\%), 85\% (range, 73\%-93\%), 50\% (range, 27\%-73\%), 95\% (range, 84\%$99 \%$ ), and $84 \%$ (range, 73\%-90\%). The inter-rater agreement was good for evaluating FPCT with respect to intracranial bleeding $(\kappa=0.76 ; 95 \%$ CI, $0.58-0.94)$ and BBB disruption $(\kappa=0.68$; 95\% CI, 0.48-0.87).

\section{Attenuation Measurement}

If one divided FPCT hyperattenuation into SAH, ICB, and BBB disruptions, the median values of maximum attenuation measurements in true-positive and false-positive regions were 159 versus 223 ( $n=6$ versus $4 ; P=.524), 375$ versus 678 ( $n=2$ versus 2; $P=.833)$, and 163 versus $114(n=8$ versus $6 ; P=.103)$, respectively. A significant difference could not be found in any of the 3 subgroups.

\section{DISCUSSION}

Flat panel detector-based CT is known to be a very useful tool for the detection or exclusion of peri-interventional complications. ${ }^{6}$ It provides cross-sectional images of the brain without the necessity of transferring patients from the angiography suite to a conventional CT facility. ${ }^{4,15}$

In a swine brain model, Arakawa et $\mathrm{al}^{5}$ were able to detect intracranial hematomas with a hematocrit level of 20\%. Symptomatic intracerebral hemorrhage still represents the most feared complication of treatment with intravenous tPA. While hemorrhagic infarction may be a clinically irrelevant epiphenomenon of ischemic damage and reperfusion, ${ }^{16,17}$ parenchymal hematoma was hypothesized to be related to the biologic effects of tPA and other pre-existing pathologic conditions associated with a worse outcome and higher mortality. ${ }^{18,19}$ Accordingly, the severity and duration of ischemia were found to be predictors of the type of hemorrhagic transformation, but not parenchymal hematoma type after IV thrombolysis. ${ }^{20}$ In the present study, acute stroke therapy consisted of endovascular intervention in all patients. Instead of exclusive IV thrombolytic drug treatment, intra-arterial urokinase or the Solitaire FR thrombectomy system (Covidien, Irvine, California) were applied with or without IV rtPA bridging therapy. The Solitaire endovascular device has proved to be a valid tool in the treatment of acute ischemic stroke, providing effective and relatively safe arterial recanalization. ${ }^{11,12,21,22}$

In our study of FPCT after endovascular stroke treatmentwith IV bridging thrombolysis in 10\%, intra-arterial thrombolysis in $56 \%$, and mechanical thrombectomy in $81 \%$ of patients - an intracranial hematoma was present in $16 \%$ of all cases. While most ICB ( $5 \%$ of patients) was found to be a clinically less relevant hemorrhagic transformation, parenchymal hematoma was a rare incident (4\%); however, it was combined with SAH in all 3 cases. ${ }^{13}$ Subarachnoid hemorrhage was still found in $11 \%$ of patients.

For the detection of intracranial bleeding by FPCT, the positive predictive value in our series of 73 patients was remarkably low (44\%). In contrast to that, we were able to exclude hemorrhage in the interventional unit with a negative predictive value of $91 \%$.

Inferior or, rarely, insufficient image quality in the posterior fossa was shown to be a drawback of FPCT, which may become relevant in macroembolic stroke of the posterior circulation. However, infratentorial blood was detected in only 2 patients in our series with peri-interventional extravasation during recanalization of 1 of their middle cerebral arteries, and it was combined with supratentorial hemorrhage in both cases.

Albeit in a small number of relevant cases, attenuation measurements did not seem to be useful for the differentiation of postinterventional hyperattenuation in FPCT.

Following diagnostic cerebral angiography and interventional procedures, immediate brain CT findings may reveal various patterns of abnormal contrast enhancement, with or without neurologic deficits. ${ }^{8,9,23-25}$ Such findings, which are believed to result from some degree of BBB disruption or a change in vascular permeability, can mimic SAH, intraventricular hemorrhage, or socalled hyperintense acute reperfusion marker on fluid-attenuated inversion recovery MR imaging ${ }^{25-27}$ and may cause unnecessary delay in anticoagulant/antiaggregant treatment. After apparently uneventful endovascular coiling of intracranial aneurysms, a sig- 
nificant relationship was reported between the occurrence of transient cortical hyperattenuation and the amount of contrast material used per kilogram of body weight, the microcatheter time, the number of balloon inflations, the total time of balloon inflation, BBB changes following temporary and permanent ischemia, SAH, more advanced patient age, spontaneous hypertension as a result of transient ischemia, and IV heparin.

These previous findings may be transferred to transarterial stroke interventions with reservation. An inverse relation was reported between transient cortical hyperattenuation and the time elapsed until the CT was performed. ${ }^{8}$ In this context, a time interval of, on average, 17.2 hours (range, $0.6-45.9$ hours) between FPCT and CCT must be considered for the interpretation of our results, with FPCT performed immediately postintervention. This latency period should be appropriate for intracranial hemorrhage to be confirmed by the criterion standard. On the other hand, it may be hypothesized that transient hyperattenuation due to BBB disruptions-as observed in $16 \%$ of CCT follow-up scans-may resolve earlier than bleeding-related hyperattenuation. With only moderate agreement between FPCT and CCT, a positive predictive value of not more than $50 \%$ contradicted a negative predictive value of $95 \%$. Although BBB disruption was suspected in FPCT much more often than in CCT, it did not turn out to be hemorrhage in any case of our series.

In the study published by Baik et al, ${ }^{9} \mathrm{MR}$ imaging did not reveal any corresponding abnormalities and the abnormal CT findings had partially or totally resolved on 24-hour follow-up CT scans. The breakdown of the BBB in the presence of iodine is known to be related to the osmolality and chemical structure of the contrast medium, the route of administration, and the speed of injection. ${ }^{28,29}$ Besides SAH, different types of abnormal hyperattenuation have been reported immediately after embolization of cerebral aneurysms, such as subarachnoid hyperattenuation, cortical hyperattenuation, and intraventricular and striatal contrast enhancement. ${ }^{9,15}$ This finding may be attributable to the fact that intravascular contrast media potentially passes the bloodbrain interface, the blood-CSF interface, and/or the brain-CSF interface. ${ }^{30}$ Following recanalization of the middle cerebral artery, incomplete striatal hyperattenuation was the most common imaging pattern attributable to BBB disruption in our study.

Potentially, a major limitation of this study is the latency between FPCT and CCT because early BBB disruption may be a precursor of symptomatic intracerebral hemorrhage. ${ }^{23,24}$ However, this progression has not been the case in any of our patients. While FPCT - the method under investigation — served as an instant postinterventional examination, there is a widely accepted recommendation for follow-up CCT, serving as the criterion standard, to be performed approximately 24 hours after acute stroke treatment. The dual-energy mode, which had been reported to be of great value in this context, was not applied during CCT acquisition. ${ }^{31}$ Second, a few patients with stroke had to be excluded from this retrospective study due to missing FPCT scans or unacceptably long follow-up periods - both caused by individual situations in clinical routine. Third, our study is based on a relatively inhomogeneous population as far as the mode of acute stroke treatment is concerned with intra-arterial thrombolysis or mechanical thrombectomy being the mainstay of therapy. Finally,
FPCT has considerable limited image quality below the tentorium. Even though playing a minor role in macroembolic stroke of the anterior circulation, limited image quality below the tentorium may potentially be relevant to patients after recanalization of the basilar artery. In our opinion, the qualitative image characteristics of both FPCT units were comparable for the objective of this study.

\section{CONCLUSIONS}

Severe bleeding complications seem to be rare after endovascular treatment of macroembolic stroke and may be associated with iatrogenic perforation of an intracranial artery. Flat panel CT in the immediate follow-up of endovascular stroke treatment was able to exclude intracranial hemorrhage with a high negative predictive value. Future studies should evaluate the predictive value of BBB disruptions in FPCT for the development of relevant hematomas.

Disclosures: Thomas Kau-UNRELATED: Travel/Accommodations/Meeting Expenses Unrelated to Activities Listed: Philips, Siemens, Guerbet, Bayer-Schering, Bracco, ev3, Boston Scientific. Michael Hauser-UNRELATED: Travel/Accommodations/Meeting Expenses Unrelated to Activities Listed: Medtronic (Congress), Covidien (Congress). Sonja M. Obmann—UNRELATED: Payment for Lectures (including service on Speakers Bureaus): Boehringer-Ingelheim, Pfizer, Lilly. Jörge R. WeberUNRELATED: Consultancy: Biogen Idec, Novartis, Boehringer-Ingelheim, Bayer, Sanofi, Merck, Comments: consultancy and expert opinions, Payment for Development of Educational Presentations: Biogen, Idec Austria, Novartis Austria, Comments: The presentation for Biogen Idec was only for internal use within the company. Klaus A. Hausegger-UNRELATED: Board Membership: Cardiovascular and Interventional Radiological Society of Europe, Payment for Lectures (including service on Speakers Bureaus): Fachhochschule Kärnten.

\section{REFERENCES}

1. Jaffray DA, Siewerdsen JH. Cone-beam computed tomography with a flat-panel imager: initial performance characterization. Med Phys 2000;27:1311-23

2. Gupta R, Cheung AC, Bartling SH, et al. Flat-panel volume CT: fundamental principles, technology, and applications. Radiographics 2008;28:2009-22

3. Struffert T, Doerfler A. Flat-detector computed tomography in diagnostic and interventional neuroradiology [German]. Radiolog 2009;49:820-29

4. Hausegger KA, Fürstner M, Hauser M, et al. Clinical application of flat-panel CT in the angio suite [German]. Rofo 2011;183:1116-22

5. Arakawa H, Marks MP, Do HM, et al. Experimental study of intracranial hematoma detection with flat panel detector C-arm CT. AJNR Am J Neuroradiol 2008;29:766-72

6. Psychogios MN, Buhk JH, Schramm P, et al. Feasibility of angiographic $\mathrm{CT}$ in peri-interventional diagnostic imaging: a comparative study with multidetector CT. AJNR Am J Neuroradiol 2010;31:1226-31

7. Uchiyama Y, Abe T, Tanaka N, et al. Factors contributing to bloodbrain barrier disruption following intracarotid injection of nonionic iodinated contrast medium for cerebral angiography: experimental study in rabbits. Radiat Med 2006;24:3212-16

8. Ozturk A, Saatci I, Pamuk AG, et al. Focal increased cortical density in immediate postembolization CT scans of patients with intracranial aneurysms. AJNR Am J Neuroradiol 2006;27:1866-75

9. Baik SK, Kim YS, Lee HJ, et al. Immediate CT findings following embolization of cerebral aneurysms: suggestion of blood-brain barrier or vascular permeability change. Neuroradiology 2008;50:259-66

10. Mullen MT, Pisapia JM, Tilwa S, et al. Systematic review of outcome after ischemic stroke due to anterior circulation occlusion treated 
with intravenous, intra-arterial, or combined intravenous + intraarterial thrombolysis. Stroke 2012;43:2350-55

11. Mordasini P, Brekenfeld C, Byrne JV, et al. Technical feasibility and application of mechanical thrombectomy with the Solitaire FR revascularization device in acute basilar artery occlusion. AJNR Am J Neuroradiol 2013;34:159-63

12. Pereira VM, Gralla J, Davalos A, et al. Prospective, multicenter, single-arm study of mechanical thrombectomy using Solitaire flow restoration in acute ischemic stroke. Stroke 2013;44:2802-07

13. Fiorelli M, Bastianello S, von Kummer R, et al. Hemorrhagic transformation within 36 hours of a cerebral infarct: relationships with early clinical deterioration and 3-month outcome in the European Cooperative Acute Stroke Study I (ECASS I) cohort. Stroke 1999; 30:2280-84

14. Fisher CM, Kistlet JP, Davis JM. Relation of cerebral vasospasm to subarachnoid hemorrhage visualized by computerized tomographic scanning. Neurosurgery 1980;6:1-9

15. Shinohara Y, Sakamoto M, Takeuchi H, et al. Subarachnoid hyperattenuation on flat panel detector-based conebeam CT immediately after uneventful coil embolization of unruptured intracranial aneurysms. AJNR Am J Neuroradiol 2013;34:577-82

16. Molina CA, Alvarez-Sabin J, Montaner J, et al. Thrombolysis-related hemorrhagic infarction: a marker of early reperfusion, reduced infarct size, and improved outcome in patients with proximal middle cerebral artery occlusion. Stroke 2002;33:1551-56

17. von Kummer R. Brain hemorrhage after thrombolysis: good or bad? Stroke 2002;33:1446-47

18. Larrue V, von Kummer RR, Muller A, et al. Risk factors for severe hemorrhagic transformation in ischemic stroke patients treated with recombinant tissue plasminogen activator: a secondary analysis of the European-Australasian Acute Stroke Study (ECASS II). Stroke 2001;32:438-41

19. Berger C, Fiorelli M, Steiner T, et al. Hemorrhagic transformation of ischemic brain tissue: asymptomatic or symptomatic? Stroke 2001; 32:1330-35

20. Thomalla G, Sobesky J, Köhrmann M, et al. Two tales: hemorrhagic transformation but not parenchymal hemorrhage after thrombolysis is related to severity and duration of ischemia: MRI study of acute stroke patients treated with intravenous tissue plasminogen activator within 6 hours. Stroke 2007;38:313-18
21. Mokin M, Dumont TM, Veznedaroglu E, et al. Solitaire flow restoration thrombectomy for acute ischemic stroke: retrospective multicenter analysis of early postmarket experience after FDA approval. Neurosurgery 2013;73:19-25

22. Yoon YH, Yoon W, Jung MY, et al. Outcome of mechanical thrombectomy with Solitaire stent as first-line intra-arterial treatment in intracranial internal carotid artery occlusion. Neuroradiology 2013; 55:999-1005

23. Hjort N, Wu O, Ashkanian M, et al. MRI detection of early bloodbrain barrier disruption: parenchymal enhancement predicts focal hemorrhagic transformation after thrombolysis. Stroke 2008;39: 1025-28

24. Kastrup A, Gröschel K, Ringer TM, et al. Early disruption of the blood-brain barrier after thrombolytic therapy predicts hemorrhage in patients with acute stroke. Stroke 2008;39:2385-87

25. Köhrmann M, Struffert T, Frenzel T, et al. The hyperintense acute reperfusion marker on fluid-attenuated inversion recovery magnetic resonance imaging is caused by gadolinium in the cerebrospinal fluid. Stroke 2012;43:259-61

26. Morris JM, Miller GM. Increased signal in the subarachnoid space on fluid-attenuated inversion recovery imaging associated with the clearance dynamics of gadolinium chelate: a potential diagnostic pitfall. AJNR Am J Neuroradiol 2007;28:1964-67

27. Rozanski M, Ebinger M, Schmidt WU, et al. Hyperintense acute reperfusion marker on FLAIR is not associated with early haemorrhagic transformation in the elderly. Eur Radiol 2010;20:2990-96

28. Wilson AJ, Evill CA, Sage MR. Effects of nonionic contrast media on the blood brain barrier: osmolality versus chemotoxicity. Invest $R a$ diol 1991;26:1091-94

29. Kuhn MJ, Burk TJ, Powell FC. Unilateral cerebral cortical and basal ganglia enhancement following overdosage of nonionic contrast media. Comput Med Imaging Graph 1995;19:307-11

30. Sage MR, Wilson AJ, Scroop R. Contrast media and the brain: the basis of CT and MR imaging enhancement. Neuroimaging Clin NAm 1998;8:695-707

31. Phan CM, Yoo AJ, Hirsch JA, et al. Differentiation of hemorrhage from iodinated contrast in different intracranial compartments using dual-energy head CT. AJNR Am J Neuroradiol 2012;33:1088-94 